\title{
Resistenzen gegen Zweitlinienmedikamente bei Migranten mit multiresistenter Tuberkulose in der Region Berlin*
}

\author{
Resistance to Second-Line Drugs in Migrants with Multidrug-Resistant \\ Tuberculosis in the Berlin Region
}

Autoren

Institute
R. Otto-Knapp ${ }^{1,2}$, L. Bös ${ }^{2}$, N. Schönfeld ${ }^{1}$, S. Wagner ${ }^{3}$, A. K. Starzacher ${ }^{1,4}$, T. Weiss ${ }^{1,4}$, S. Vesenbeckh ${ }^{1,4}$, G. Glaser-Paschke ${ }^{4}$, H. Mauch ${ }^{3}$, H. Rüssmann ${ }^{3}$, T. T. Bauer ${ }^{1,2}$

${ }^{1}$ Lungenklinik Heckeshorn, HELIOS Klinikum Emil von Behring, Berlin

${ }^{2}$ Deutsches Zentralkomitee zur Bekämpfung der Tuberkulose (DZK), Berlin

${ }^{3}$ Institut für Mikrobiologie, Immunologie und Laboratoriumsmedizin, HELIOS Klinikum Emil von Behring, Berlin

${ }^{4}$ Zentrum für tuberkulosekranke und -gefährdete Menschen, Berlin eingereicht 12.4 .2014 akzeptiert nach Revision 12.5.2014

Bibliografie

DOI http://dx.doi.org/

$10.1055 / \mathrm{s}-0034-1377226$

Pneumologie 2014; 68: 496-500

(c) Georg Thieme Verlag KG

Stuttgart · New York

ISSN 0934-8387

Korrespondenzadresse Dr. Ralf Otto-Knapp, M. Sc.

Deutsches Zentralkomitee zur Bekämpfung der Tuberkulose (DZK)

Haus Q, Walterhöferstraße 11 14165 Berlin

rotto-knapp@dzk-tuberkulose.de

\section{Zusammenfassung}

$\nabla$

Die kalkulierte Behandlung der multiresistenten (MDR) Tuberkulose (TB) nach molekularer Schnelltestung wird durch die mehrwöchige Unkenntnis der Empfindlichkeit gegen Zweitlinienmedikamente erschwert. Durch die Berücksichtigung regionaler Häufungen von Resistenzen bei Migranten könnte die kalkulierte Behandlung mit Zweitlinienmedikamenten zielgerichteter erfolgen.

Von 2008 bis 2013 wurden retrospektiv die Ergebnisse der kulturellen Empfindlichkeitstestung aller eingesendeten Mykobakterienstämme des Instituts für Mikrobiologie, das mit der Lungenklinik Heckeshorn zusammenarbeitet, analysiert und auf regionale Unterschiede untersucht.

Unter den Proben fanden sich 39 Mycobacterium tuberculosis-Stämme mit Multiresistenz. Als Zweitrangmedikamente wurden Linezolid (97\%), Clofazimin (95\%), Cycloserin (95\%), Capreomycin (90\%), P-Aminosalicylsäure (82\%), Moxifloxacin (79\%) und Amikacin (79\%) bei mehr als der Hälfte der Stämme empfindlich getestet. Der Anteil der empfindlichen Stämme war niedriger für Pyrazinamid (44\%), Ethambutol (28\%), Protionamid (15\%), Rifabutin (8\%) und Streptomycin (8\%). Bei den Mykobakterienstämmen aus Tschetschenien $(n=14)$ unterschieden sich die Empfindlichkeiten gegen Amikacin (57\%) und Protionamid (36\%) signifikant von denen aus anderen Regionen.

Die regional unterschiedlich ausgeprägten Empfindlichkeiten gegen Zweitlinienmedikamente legen nahe, dass bei der Wahl der kalkulierten Initialtherapie von MDR TB-Patienten mit Migrationshintergrund differenziert vorgegangen werden muss.

\section{Abstract \\ $\nabla$}

The empiric therapy of multidrug-resistant (MDR) tuberculosis (TB) after rapid molecular testing is rendered difficult by an often several weeks-long period of uncertainty, because results of susceptibility testing for second-line TB drugs are pending. The analysis of regional resistance patterns could lead to a more targeted empiric treatment for migrants depending on their country of origin. The results of the susceptibility testing from 2008 to 2013 of all mycobacteria sent to the Institute of Microbiology, working with the department of Pneumology, Heckeshorn Lung Clinic, Berlin, were reanalysed and tested for regional differences.

We found 39 multidrug-resistant Mycobacterium tuberculosis strains among the examined strains. More than half of these strains tested susceptible to the following second line drugs namely, linezolid $(97 \%)$, clofazimine (95\%), cycloserine (95\%), capreomycin (90\%), p-aminosalicylic acid (82\%), moxifloxacin (79\%) and amikacin (79\%). The proportion of strains susceptible to pyrazinamide (44\%), ethambutol (28\%), prothionamide (15\%), rifabutin (8\%) and streptomycin (8\%) was lower. The mycobacterial cultures of the Chechen patients $(n=14)$ showed significantly different susceptibilities to amikacin (57\%) and prothionamide (36\%) compared to the strains from migrants of other regions.

In this study, the regional differences in mycobacterial susceptibility to second line drugs suggest that the initial MDR TB therapy of migrants should be tailored to their country of origin.

\footnotetext{
* Die Auswertung wurde durch die Oskar-Helene-Heim Stiftung unterstützt.
} 


\begin{tabular}{|c|c|c|c|}
\hline & $\begin{array}{l}\text { Gesamt } \\
(n=39)\end{array}$ & $\begin{array}{l}\text { Tschetschenen } \\
(n=14)\end{array}$ & $\begin{array}{l}\text { Nicht-Tschetschenen } \\
(n=25)\end{array}$ \\
\hline Alter, Median (Spannweite) & $35(19-76)$ & $38(25-54)$ & $35(19-76)$ \\
\hline \multicolumn{4}{|l|}{ Geschlecht } \\
\hline weiblich n(\%) & $13(33 \%)$ & $6(43 \%)$ & $7(28 \%)$ \\
\hline männlich n (\%) & $26(67 \%)$ & $8(57 \%)$ & $18(72 \%)$ \\
\hline \multicolumn{4}{|l|}{ kultureller Nachweis aus: } \\
\hline Sputum n(\%) & $25(64 \%)$ & $10(71 \%)$ & $15(60 \%)$ \\
\hline Bronchialsekret n(\%) & $4(10 \%)$ & $1(7 \%)$ & $3(28 \%)$ \\
\hline extrapulmonalen Materialien n (\%) & $10(26 \%)$ & $3(22 \%)$ & $7(12 \%)$ \\
\hline Mortalität n (\%) & $4(10 \%)$ & $2(14 \%)$ & $2(8 \%)$ \\
\hline
\end{tabular}

Tab. 1 Demografische Daten, diagnostisches Material zur Kulturgewinnung und Mortalität.

\section{Einleitung}

\section{$\nabla$}

Die multiresistente (MDR) Tuberkulose (TB) wird zunehmend als globales Problem wahrgenommen. Neben schlechteren Behandlungsergebnissen mit erhöhter Mortalität führt sie auch durch höhere Behandlungskosten zu einer erheblichen Mehrbelastung des Gesundheitssystems [1]. In der WHO-Region Osteuropa stieg 2012 der Anteil der MDR TB an den Neudiagnosen auf über 20\%. Bei vorbehandelten Patienten wurde sogar ein Anteil von über 50\% MDR TB beobachtet [2]. In Deutschland liegt der Anteil der MDR TB nahezu gleichbleibend bei $2 \%$, wobei Patienten ausländischer Herkunft, die vorbehandelt wurden, zu etwa $16 \%$ betroffen sind [3]. Jede zweite TB-Neudiagnose betrifft einen Patienten, der außerhalb Deutschlands geboren ist. Aus den Daten des Robert Koch-Instituts (RKI) geht auch hervor, dass Patienten mit Migrationshintergrund in Deutschland von der MDR TB deutlich häufiger betroffen sind.

Molekularbiologische Schnelltests werden heute angewendet, um innerhalb kurzer Zeit Informationen über die Empfindlichkeit des Tuberkulose-Erregers gegen Isoniazid (INH) und Rifampicin (RMP) zu erhalten. Molekulare Schnelltests gegen einige Zweitlinienmedikamente werden derzeit erprobt, sind aber noch nicht weitreichend verfügbar. Die kulturelle Resistenztestung für Medikamente der zweiten Linie ist teilweise als experimentell anzusehen und setzt spezialisiertes Personal sowie technische Ressourcen voraus. Sie benötigt je nach Methode und Wachstumsgeschwindigkeit 2 bis 10 Wochen. Die Behandlung einer durch Schnelltests diagnostizierten MDR-Tuberkulose muss daher kalkuliert erfolgen, bis die Ergebnisse der Resistenztestung verfügbar sind. Die Wahl einer geeigneten Therapie ist in dieser kritischen Phase schwierig, da nur wenige Daten zur Häufigkeit von Resistenzen gegen Zweitlinienmedikamente zur Verfügung stehen.

Die kalkulierte Behandlung orientiert sich an den WHO-Empfehlungen zur Behandlung der MDR TB [4], welche auch die Grundlage der deutschen Empfehlungen des DZK bilden [5]. Die WHOEmpfehlungen entstehen im Wesentlichen durch die Auswertung weltweit akkumulierter Daten. Regionale Unterschiede in der Erregerempfindlichkeit konnten hierbei nur unzureichend Berücksichtigung finden. Die Wirksamkeit der bei der kalkulierten Behandlung zum Einsatz kommenden Zweitlinienmedikamente ist daher im Einzelfall schwer vorhersehbar. Ohne den Schutz durch wirksame Kombinationspartner besteht jedoch die Gefahr, dass sich weitere Resistenzen gegen die initial noch wirksamen Medikamente entwickeln [8].

Ziel unserer retrospektiven Datenanalyse war es, durch die Auswertung der Medikamentenempfindlichkeiten bei MDR TBStämmen unseres Zentrums einen Eindruck über die Resistenzmuster relevanter Herkunftsregionen zu gewinnen und regionale Unterschiede herauszuarbeiten.

\section{Methoden \\ $\nabla$}

Die Ergebnisse der Empfindlichkeitstestung aller zwischen Januar 2008 und Dezember 2013 im mikrobiologischen Labor des HELIOS Klinikums Emil von Behring auf Mykobakterien untersuchten Proben wurden retrospektiv ausgewertet. Die Dokumentation der Daten erfolgte durch das Labor-Informationssystem Dorner M/Lab, die retrospektive Auswertung über HybaseKlinik. Informationen zur Mortalität wurden vom zuständigen Berliner Gesundheitsamt (Zentrum für tuberkulosekranke und -gefährdete Menschen) eingeholt. Die Empfindlichkeit der Mycobacterium tuberculosis-Stämme wurde mittels Dilutionsmethode auf Middlebrook 7H10 Agar untersucht. In Anlehnung an die Richtlinien des Clinical and Laboratory Standards Institute (CLSI) wurden folgende kritische Konzentrationen verwendet: Amikacin $4 \mu \mathrm{g} / \mathrm{ml}$, Capreomycin $10 \mu \mathrm{g} / \mathrm{ml}$, Clofazimin $2 \mu \mathrm{g} / \mathrm{ml}$, Cycloserin $30 \mu \mathrm{g} / \mathrm{ml}$, Ethambutol $5 \mu \mathrm{g} / \mathrm{ml}$, Linezolid $2 \mu \mathrm{g} / \mathrm{ml}$, Moxifloxacin $0,5 \mu \mathrm{g} / \mathrm{ml}$, P-Aminosalicylsäure $2 \mu \mathrm{g} / \mathrm{ml}$, Protionamid 2,5 $\mu \mathrm{g} / \mathrm{ml}$, Rifabutin $1 \mu \mathrm{g} / \mathrm{ml}$ und Streptomycin $2,5 \mu \mathrm{g} / \mathrm{ml}$ [6]. Zum statistischen Vergleich der Resistenzen unterschiedlicher Regionen wurde der Fisher's Exact Test verwendet.

Alle für diese Studie verwendeten Daten wurden im Rahmen der Routinediagnostik und -behandlung erhoben. Die zu den Tuberkulosekulturen gehörigen Patientendaten wurden anonymisiert ausgewertet, sodass wir auf ein schriftliches Einverständnis verzichten konnten.

\section{Ergebnisse}

Im Studienzeitraum wurden insgesamt 39 multiresistente Mycobacterium tuberculosis-Stämme nachgewiesen. Bei Therapiebeginn waren die MDR TB-Patienten im Median 35 (Spannweite 19-76) Jahre alt, 33\% waren weiblich. Bei allen Patienten wurde ein HIV-Test dokumentiert, dieser fiel bei einem Patienten (2,6\%) positiv aus. Die Diagnose einer Tuberkulose wurde bei 25 Patienten $(64 \%)$ aus dem Sputum und bei 4 Patienten (10\%) aus Bronchialsekret gestellt. Die Mortalität betrug bis zum Abschluss der Beobachtungen mit 4 verstorbenen von 39 MDR TB-Patienten $10 \%$ und betraf keinen XDR-Patienten ( Tab. 1).

Die Patienten stammten überwiegend aus dem russischen Staatsgebiet $(n=25)$, darunter 11 Patienten (28\%) aus Russland und 14 Patienten (36\%) aus Tschetschenien. 3 Patienten waren gebürtig aus Litauen (8\%), 2 aus Kasachstan (5\%), jeweils ein Patient (2,6\%) war in Afghanistan, Armenien, Bulgarien, Deutschland, Indien, Kirgistan, Kurdistan, Rumänien und Serbien geboren. 


\begin{tabular}{|lcclccc|} 
& $\begin{array}{l}\text { Gesamt } \\
(\mathbf{n = 3 9 )}\end{array}$ & $\%$ & $\begin{array}{l}\text { Tschetschenen } \\
(\mathbf{n = 1 4 )}\end{array}$ & $\%$ & $\begin{array}{l}\text { Nicht-Tschetschenen } \\
(\mathbf{n = 2 5 )}\end{array}$ & \% \\
\hline Jegliche & 38 & 97 & 14 & 100 & 24 & 96 \\
\hline Injizierbare Medikamente $^{1}$ & 9 & 23 & 6 & 43 & 3 & 12 \\
\hline Fluorchinolone $^{2}$ & 8 & 21 & 2 & 14 & 6 & 24 \\
\hline XDR-TB $^{3}$ & 2 & 5 & 2 & 14 & 0 & 0 \\
\hline
\end{tabular}

Tab. 2 Resistenzen gegen Zweitlinienmedikamente bei MDR-Tuberkulosestämmen der Lungenklinik Heckeshorn.

getestet: Amikacin, Capreomycin

2 getestet: Moxifloxacin

${ }^{3} \mathrm{XDR}-\mathrm{TB}=$ extensiv resistente Tuberkulose

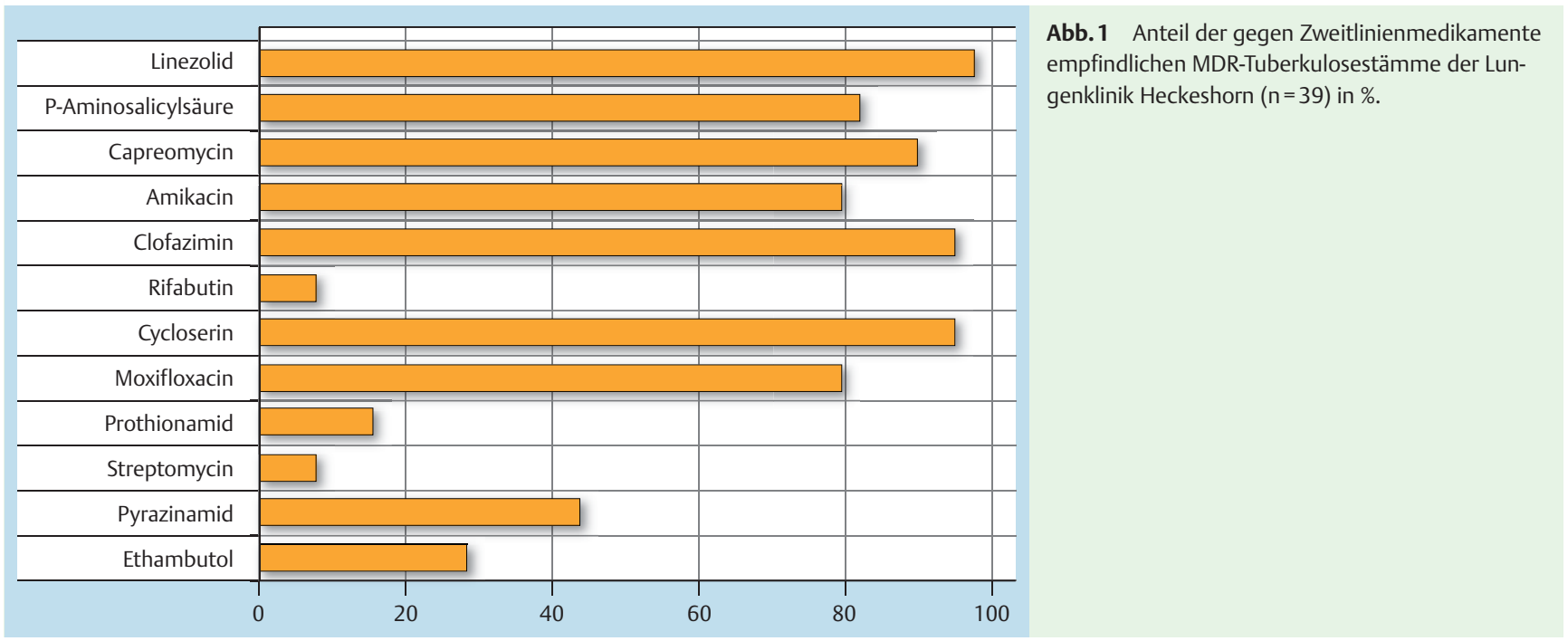

Eine Resistenz gegen zumindest eines der untersuchten Zweitrangmedikamente (Amikacin, Capreomycin, Clofazimin, Cycloserin, Linezolid, Moxifloxacin, P-Aminosalicylsäure, Protionamid, Rifabutin) wurde bei $97 \%$ der Stämme festgestellt. Eine Resistenz gegen zumindest ein injizierbares Medikament der zweiten Linie (Capreomycin und Amikacin) bestand in 23\% der Fälle, dieser Anteil war bei Stämmen aus Tschetschenien mit $43 \%$ signifikant höher. Eine Resistenz gegen Fluorchinolone (Moxifloxacin) wurde in $21 \%$ der Fälle dokumentiert. Die Definition einer extensiv resistenten (XDR) TB mit Resistenzen gegen INH, RMP, ein Fluorchinolon und ein injizierbares Medikament traf bei 5\% der Stämme zu ( Tab.2).

Von den 39 MDR TB-Stämmen wurden Linezolid (sensibel: 97\%), Clofazimin (sensibel: 95\%), Cycloserin (sensibel: 95\%), Capreomycin (sensibel: 90\%), P-Aminosalicylsäure (sensibel: 82\%), Moxifloxacin (sensibel: 79\%) und Amikacin (sensibel: 79\%) bei über der Hälfte der Stämme empfindlich getestet. Demgegenüber fanden sich weniger Stämme mit Empfindlichkeit gegen Pyrazinamid (sensibel: 44\%), Ethambutol (sensibel: 28\%), Protionamid (sensibel: 15\%), Rifabutin (sensibel: $8 \%$ ) und Streptomycin (sensibel: $8 \%)($ Abb.1).

Im Vergleich zu den restlichen Patienten $(\mathrm{n}=25)$ waren signifikante Unterschiede in der Region Tschetschenien $(n=14)$ hinsichtlich der Empfindlichkeit der MDR-Tuberkulosestämme gegen Amikacin und Protionamid auffällig. Die tschetschenischen MDR TB-Stämme waren mit 57\% gegenüber 92\% signifikant $(p=0,0161)$ seltener empfindlich gegen Amikacin. Protionamid dagegen wurde mit $36 \%$ bei den Tschetschenen gegenüber $4 \%$ bei den nicht-Tschetschenen signifikant $(p=0,0163)$ häufiger empfindlich getestet ( $\bullet$ Abb.2a u. $\bullet$ Abb.2b). Demografische Daten, Diagnostik und Mortalität beider Gruppen unterschieden sich nicht signifikant ( Tab. 1).

\section{Diskussion \\ $\nabla$}

Unsere retrospektive Auswertung gibt Aufschluss über die Häufigkeit von zusätzlichen Medikamentenresistenzen bei $38 \mathrm{Mi}$ granten und einem deutschen Patienten mit MDR-Tuberkulose am Beispiel eines regionalen Zentrums (Lungenklinik Heckeshorn, Berlin). Medikamentenempfindlichkeiten, die sich von anderen Herkunftsregionen signifikant unterschieden, wurden bei den tschetschenischen Patienten gefunden. Unterschiedliche Resistenzmuster zeigten sich auch für andere Regionen, diese erreichten jedoch - auch aufgrund der geringen Fallzahlen - kein Signifikanzniveau.

In der multizentrischen PETTS (Preserving Effective TB Treatment Study) Studie unter Mitwirkung der Centers for Disease Control (CDC) wurden 1278 MDR TB-Isolate aus 8 Ländern unterschiedlicher Regionen (Estland, Lettland, Peru, Philippinen, Russland, Südafrika, Südkorea und Thailand) auf Resistenzen gegen Zweitrangmedikamente untersucht [7]. In dieser prospektiven Studie wurde bei $43,7 \%$ der MDR TB-Patienten zumindest eine Resistenz gegen ein Zweitlinienmedikament gefunden. Eine Resistenz gegen ein injizierbares Medikament (Kanamycin, Capreomycin, Amikacin) wurde in 20,0\% der Fälle gesehen und eine Fluorchinolon-Resistenz (Ofloxacin und Ciprofloxacin) in 12,9\% der MDR TB-Fälle.

Der Anteil der Resistenzen gegen injizierbare Zweitlinienmedikamente fiel in unserer Studie in vergleichbarer Höhe aus. Kanamycin wurde allerdings nicht routinemäßig getestet. Kreuzresistenzen zwischen den drei Substanzen sind bekannt [8]. Die Wertigkeit der Kreuzresistenzen und die molekulargenetischen Hintergründe sind Gegenstand aktueller Untersuchungen [9]. Eine ergänzende Testung von Kanamycin hätte in unserer Studie ver- 


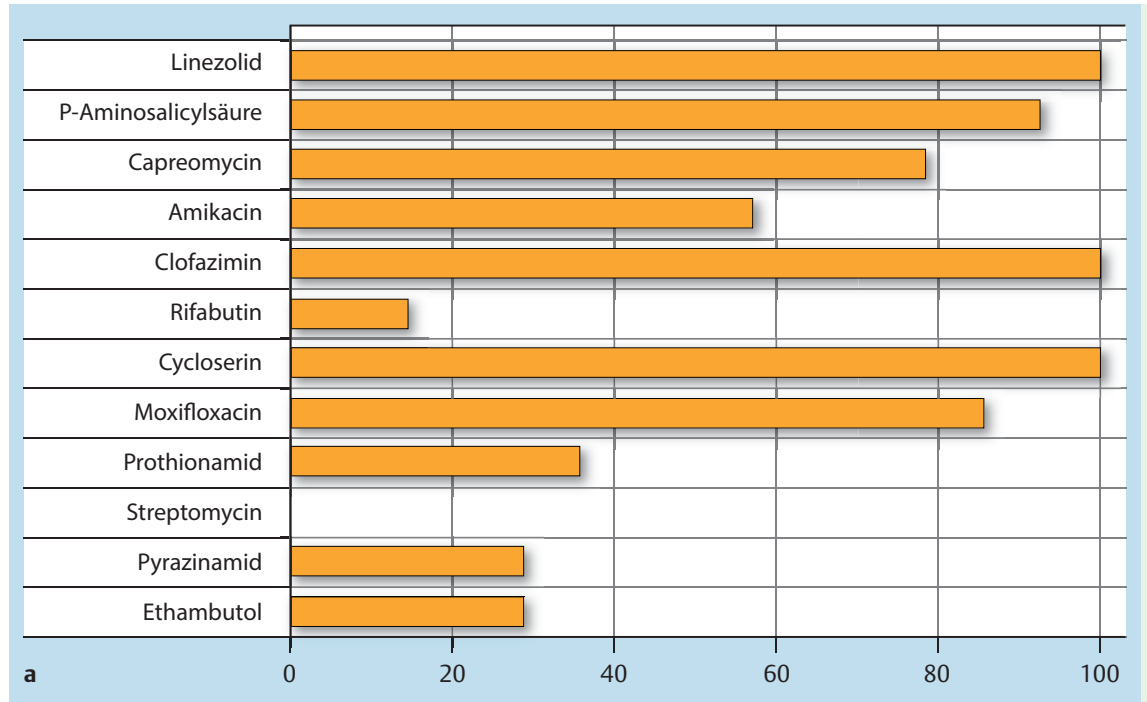

Abb.2 Anteil der gegen Zweitlinienmedikamente empfindlichen MDR-Tuberkulosestämme der Lungenklinik Heckeshorn in \%. a Tschetschenen $(n=14)$. $\mathbf{b}$ nicht-Tschetschenen $(n=25)$.

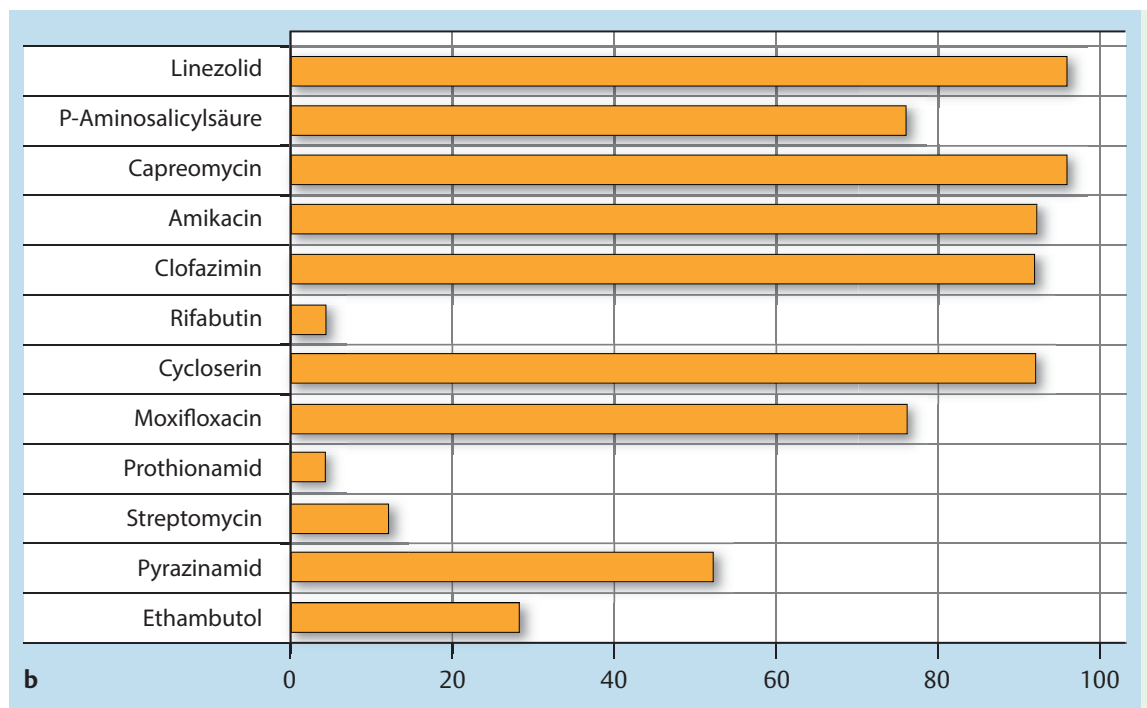

mutlich den Anteil an Resistenzen gegen injizierbare Medikamente erhöht.

Dalton u. Mitarb. fanden in Osteuropa und im Baltikum ebenfalls über 95\% Streptomycinresistenzen, während diese in allen anderen untersuchten Regionen deutlich seltener anzufinden waren [7].

In unserer Studie zeigten die Stämme aus der Region Tschetschenien einen signifikant höheren Anteil an Resistenzen gegen Amikacin. Daten der WHO [10,11] und der PETTS-Studie [7] zeigten ebenfalls relevante Unterschiede zwischen den regionalen Resistenzhäufigkeiten verschiedener Hochprävalenzregionen weltweit. Auch innerhalb Osteuropas konnten regionale Unterschiede gezeigt werden. Resistenzen gegen die injizierbaren Medikamente Capreomycin bzw. Amikacin traten in Estland/Lettland/Russland in jeweils $4,4 \% / 15,0 \% / 7,0 \%$ bzw. $23,9 \% / 35,0 \% / 16,5 \%$ der Stämme auf [7]. In Anbetracht der Toxizität dieser Medikamentengruppe wäre die Kenntnis regional hoher Resistenzraten für die kalkulierte Therapie von besonderer Relevanz.

Als Risikofaktoren für zusätzliche Medikamentenresistenzen bei MDR TB-Patienten sind vor allem die Vorbehandlung mit Zweitlinienmedikamenten und vorherige Krankenhausaufenthalte mit der Gefahr einer nosokomialen Übertragung beschrieben [12]. Soziale Indikatoren wie der Aufenthalt in Gefängnissen, Arbeits- losigkeit, Alkoholismus und Rauchen scheinen vor allem das Auftreten von Resistenzen gegen injizierbare Zweitlinienmedikamente zu beeinflussen [7]. In einer russischen Studie entwickelten $5 \%$ der MDR TB-Patienten eine XDR TB im Verlauf der Therapie, 6 Monate nach Abschluss der Therapie stieg dieser Anteil sogar auf $11 \%$ [13]. Eine weitere retrospektive russische Studie konnte die Entwicklung von 6\% XDR TB unter MDR TB-Therapie bestätigen [14]. Beide Autoren arbeiteten mangelnde Therapieadhärenz und Therapieunterbrechungen als Prädiktoren heraus. Eine Analyse der Risikofaktoren für zusätzliche Medikamentenresistenzen konnte anhand unserer Daten nicht durchgeführt werden, da die dokumentierten Informationen zu Vorbehandlung, Therapietreue und sozialen Indikatoren unvollständig waren.

Dalton u. Mitarb. diskutieren als weiteren bedeutsamen Risikofaktor für das Auftreten von zusätzlichen Resistenzen bei MDR TB-Patienten den Zeitraum der Verfügbarkeit von Zweitlinienmedikamenten in einer Region [7]. So traten in der PETTS-Studie die meisten Resistenzen in Südkorea und Russland auf, wo Zweitlinienmedikamente 20 Jahre und länger zur Verfügung standen. Die geringsten Resistenzraten waren in Thailand, den Philippinen und Peru mit 10 und weniger Jahren Verfügbarkeit beobachtet worden. Die Verfügbarkeit einer Substanz kann die Vorausset- 
zungen für die Entwicklung von Resistenzen schaffen. Informationen zur Verfügbarkeit und zum Einsatz von Zweitlinienmedikamenten in Tschetschenien liegen uns nicht vor. Es darf jedoch angenommen werden, dass in Tschetschenien als Teil der russischen Föderation Versorgungsstrukturen anzutreffen sind, die mit dieser vergleichbar sind.

Die PETTS-Studie zeigte für Ethionamid in Estland, Lettland und Russland mit 10,9\%, 23,0\% und 13,9\% Resistenzen ebenfalls deutliche regionale Unterschiede innerhalb Osteuropas [7]. Unsere Untersuchung zeigte signifikant weniger Protionamid-Resistenzen bei den tschetschenischen Stämmen (64\%), verglichen mit den übrigen Stämmen (96\%). Die Gründe hierfür sind möglicherweise in der regional schlechteren Verfügbarkeit von Protionamid zu suchen. Resistenzen gegen Thionamide (Ethionamid/ Protionamid) entwickeln sich schnell, wenn sie nicht in einer wirksamen Kombinationstherapie verabreicht werden. Es besteht eine vollständige Kreuzresistenz zwischen Ethionamid und Protionamid [8].

Der Anteil der gegen Fluorchinolone resistenten Stämme fiel in unserer Studie mit 21\% deutlich höher aus als bei Dalton u. Mitarb. [7]. Dies ist im Wesentlichen auf den hohen Anteil von Stämmen aus Osteuropa und vor allem Russland (25/39) zurückzuführen. Eine Subgruppenanalyse der PETTS-Studie zeigte bei den russischen Stämmen $(n=115)$ mit $18 \%$ ebenfalls einen deutlich höheren Anteil von Fluorchinolon-Resistenzen. Bei den Stämmen aus Estland fielen diese sogar noch deutlich höher aus (26\%) [7]. Die Empfindlichkeitstestung in der Lungenklinik Heckeshorn wurde ausschließlich für die hier bei MDR TB-Patienten verwendete Substanz Moxifloxacin durchgeführt, sodass der Anteil an Fluorchinolon-resistenten Stämmen eher unterschätzt wurde.

Die Ergebnisse unserer regionalen, monozentrischen Auswertung werden vor allem durch die geringen Fallzahlen limitiert und sind daher nicht sicher auf MDR TB-Patienten der jeweiligen Herkunftsregionen übertragbar. In unserer Studie sind tschetschenische Patienten überrepräsentiert (14/39). Die Gründe hierfür sind unklar. Da sich einige unserer MDR TB-Patienten vor Aufnahme in die Klinik gegenseitig bekannt waren, ist auch eine Clusterbildung nicht ausgeschlossen. Eine genotypische Analyse der MDR TB-Stämme wäre in diesem Zusammenhang interessant. Da die Versorgung in den jeweiligen Heimatländern teilweise unter deutlich schlechteren Bedingungen stattfindet, erscheint auch eine Zuwanderung aus gesundheitlichen Gründen möglich.

Durch regionale Resistenzmuster könnte die kalkulierte Therapie bis zum Ergebnis der kulturellen Resistenztestung verbessert werden, um möglicherweise die Entstehung weiterer Resistenzen gegen Zweitlinienmedikamente zu verhindern. Unsere Studie zeigt am Beispiel der tschetschenischen Subpopulation, dass die Kenntnis der Resistenzmuster des jeweiligen Herkunftslandes bei Patienten mit Migrationshintergrund von Bedeutung sein kann. Das in Kürze erscheinende Konsenspapier des Tuberkulosenetzwerkes TB-NET zur Behandlung der multiresistenten
Tuberkulose in Europa empfiehlt ebenfalls - wenn möglich - regionale Resistenzmuster zu berücksichtigen [15]. Weitere kontrollierte Studien an größeren MDR TB-Populationen sind notwendig, um unser Verständnis über regionale Unterschiede bei Resistenzen gegen Zweitlinienmedikamente zu verbessern und die Auswirkungen einer nach Herkunft differenzierten, kalkulierten MDR TB-Therapie auf das Therapieergebnis zu bewerten.

\section{Interessenkonflikt}

$\nabla$

Die Autoren geben an, dass kein Interessenkonflikt besteht.

\section{Literatur}

1 Diel R, Rutz S, Castell S et al. Tuberculosis: cost of illness in Germany. Eur Respir J 2012; 40: 143 -151

2 World Health Organisation. Global Tuberculosis Report. WHO/HTM/ TB/2013.11 2013

3 Robert Koch-Institut. RKI-Bericht zur Epidemiologie der Tuberkulose in Deutschland für 2012. Im Internet: www.rki.de [Stand: 24.04.2014]

4 World Health Organisation. Guidelines for the programmatic management of drug-resistant tuberculosis. WHO/HTM/TB/2011 update 2011; 6. ed.

5 Schaberg T, Bauer T, Castell S et al. Empfehlungen zur Therapie, Chemoprävention und Chemoprophylaxe der Tuberkulose im Erwachsenenund Kindesalter. Pneumologie 2012; 66: $133-171$

6 NCCLS. Susceptibility testing of mycobacteria, nocardia, and other aerobic actinomycetes. Approved standard M24-A23: vol. Wayne, PA: National Committee for Clinical Laboratory Standards; 2003

7 Dalton T, Cegielski P, Akksilp $S$ et al. Prevalence of and risk factors for resistance to second-line drugs in people with multidrug-resistant tuberculosis in eight countries: a prospective cohort study. Lancet 2012; 380: 1406-1417

8 Caminero JA, Sotgiu G, Zumla A et al. Best drug treatment for multidrugresistant and extensively drug-resistant tuberculosis. Lancet Infect Dis 2010; 10: $621-629$

9 Maus CE, Plikaytis BB, Shinnick TM. Molecular Analysis of Cross-Resistance to Capreomycin, Kanamycin, Amikacin, and Viomycin in Mycobacterium tuberculosis. Antimicrob Agents Chemother 2005; 49: $3192-3197$

10 World Health Organisation. Multidrug and extensively drug-resistant TB (M/XDR-TB). WHO/HTM/TB/2010.3. 2010

11 European Centre for Disease Prevention and Control/WHO Regional Office for Europe. Tuberculosis surveillance and monitoring in Europe. 2012. Im Internet: www.ecdc.europa.eu [Stand: 24.04.2014]

12 Andrews JR, Shah NS, Weissman D et al. Predictors of Multidrug- and Extensively Drug-Resistant Tuberculosis in a High HIV Prevalence Community. Plos One 2010; 5: 15735

13 Cavanaugh JS, Kazennyy BY, Nguyen ML et al. Outcomes and follow-up of patients treated for multidrug-resistant tuberculosis in Orel, Russia, 2002-2005. Int J Tuberc Lung Dis 2012; 16: 1069-1074

14 Shin SS, Keshavjee S, Gelmanova IY et al. Development of Extensively Drug-resistant Tuberculosis during Multidrug-resistant Tuberculosis Treatment. Am J Respir Crit Care Med 2010; 182: 426-432

15 Lange C, Abubakar I, Alffenaar JW et al. Management of patients with multidrug-resistant/extensively drug-resistant tuberculosis in Europe: a TBNET consensus statement. Eur Respir J 2014. DOI 10.1183/ 09031936.00188313 